\title{
El cuarto número del 2017 en NeuroGastroLATAM
} \section{Reviews}

Max Schmulson

Laboratorio de Hígado, Páncreas y Motilidad (HIPAM), Unidad de Investigación en Medicina Experimental, Facultad de Medicina, Universidad Nacional Autónoma de México (UNAM), Hospital General de México

En estenúmerodela revista NeuroGastroLATAM Reviews se presenta una variedad de artículos relacionados con la motilidad y los trastornos funcionales digestivos en adultos y manejo del dolor abdominal en niños.

En el primer artículo, Bilder y Goin revisan el compromiso de la motilidad desencadenado por el Tripanosoma cruzi, agente etiológico de la enfermedad de Chagas, endémica en países de nuestra región, principalmente en Sudamérica ${ }^{1,2}$. Destacan que el megaesófago y el megacolon son los principales órganos del tubo digestivo comprometidos en la fase crónica, probablemente por pérdida de células gliales y células intersticiales de $\mathrm{Cajal}^{3}$. Estudios histopatológicos de los plexos mientéricos del esófago y del colon han demostrado que en el primero estos están vascularizados, mientras que en el colon son avasculares. Esta diferencia podría explicar la mayor entrada del agente infeccioso, con el

\section{Correspondence to:}

Max Schmulson Wasserman

Laboratorio de Hígado, Páncreas y Motilidad (HIPAM)

Unidad de Investigación en Medicina Experimental

Facultad de Medicina, UNAM, Hospital General de México

Dr. Balmis, 148

Col. Doctores

C.P. 06726, Ciudad de México, México

E-mail:maxjulio@prodigy.net.mx desarrollo de ganglionitis y denervación que llevaría al desarrollo de megaesófago y cambios de acalasia o pseudoacalasia, hacienă que el megaesófago sea mucho más frecuente que el megacolon ${ }^{4}$. Bilder y Goin refierèn que en el caso de megaesófago se debe discriminar de acalasia verdadera mediante la detección de anticuerpos contra el T. cruzi Mencionan además los avances diagnósticos logrados con la manometría esofágica de ațta resolución, pero debemos esperar los resŭltados de los estudios que se encuentran en curso para evaluar esta técnica manométrica en pacientes con megaesófago por Chagăs. En cuanto al megacolon, es importante coinsiderar los antecedentes epidemiológicos del paciente para pensar en Chagas como causa y tener en cuenta la posibilidad de la péräida del reflejo rectoanal inhibitorio en la manometría anorrectal de estos pacientes. Ấ mismo, revisan el rol del sistema inmunitario en la generación de la pseudoacalasia, èn 
especial el papel de los anticuerpos monoclonales contra las estructuras neuronales y músculo liso, y los anticuerpos contra receptores muscarínicos de acetilcolina originalmente descritos en la cardiomiopatía chagásica $^{3,5,6}$.

En el segundo artículo de este número, Enrico Corazziari hace una revisión de la sensibilidad al gluten no celíaca y el síndrome de intestino irritable (SII), un tema de actualidad pero a su vez muy controvertido 7 . Por un lado, las dos entidades pueden manifestarse de manera independiente y son difíciles de diferenciar clínicamente una de otra por la ausencia de biomarcadores específicos; por otra parte, las dos entidades pueden sobreponerse ${ }^{8}$. Además, la dificultad en diferenciar la sensibilidad al gluten de aquella a los carbohidratos fermentables (FODMAP) es un factor que contribuye a la confusión, si consideramos que el trigo contiene fructanos, un FODMAP; además, la dieta baja en FODMAP parece ser más efectiva que la dieta baja en gluten, sobre todo en el SII ${ }^{9,10}$. Corazziari presenta un algoritmo práctico de diagnóstico para tratar de discriminar la sensibilidad al gluten en la dieta de aquella a los FODMAP tanto en pacientes que ya se encuentran en dieta libre de gluten como en aquellos que mantienen una dieta rica en gluten. En este algoritmo, el reto dietético doble ciego con gluten frente a placebo juega un papel importante?

Por su parte, Laura Solé presenta en el tercer artículo una revisión muy completa de la literatura de los últimos 10 años sobre los trastornos de la evacuación ${ }^{11}$. Si bien dichos trastornos se clasifican mediante los criterios de Roma IV ${ }^{12}$, requieren el uso de pruebas fisiológicas como manometría anorrectal, prueba de expulsión del balón y estudios de imagenología como defecografía convencional y por resonancia magnética $(\mathrm{RM})^{12-14}$. Desde el punto de vista de los estudios de imagen, habrá que determinar si la defecografía por RM podrá reemplazar a la convencional, puesto que parece ser superior en la detección de enteroceles, pero presenta menor tasa de detecciön para rectoceles, intususcepción y descenso perineal ${ }^{14}$. Por otra parte, en estreñimiento oø̆structivo, la RM muestra una concordancia casi perfecta con la convencional en detección anismo y prolapso rectal. Además, la aceptáción es superior por parte de los pacientes ${ }^{13}$. a etiopatogenia de estos trastornos es desconocida, si bien una tercera parte comienzan en la infancia. Se resalta la importancia del examen digital rectal en el diagnóstico de estos pacièntes, una maniobra que es poco utilizada por los médicos y sobre la cual existe poca enšeñanza en las escuelas de medicina ${ }^{15,16}$. De hecho los estudiantes de medicina realizan pocos exámenes rectales antes de graduarse, cual redunda en un mal entrenamiento en eșta parte fundamental del examen físico ${ }^{16}$. Sọ̀lé destaca el papel de la biorretroalimentaciớn como tratamiento de elección en los trastornos funcionales de la evacuación y se presenta un resumen de los estudios controlados y aleatorizados que han analizado esta terapia, donde se evidencia su superioridad sobre el place tratamiento estándar, polietilenglicol e incluso las benzodiazepinas. Si bien se desconoce el mecanismo por el cual la bioretroalimentaciŏ̉n mejora los trastornos de la evacuación, se discute la posibilidad de mejorar la comunicación del eje intestino-cerebro ${ }^{11}$.

El último artículo de este número es una actualización de los tratamientos para el dolor abdominal en niños con trastornos 
funcionales gastrointestinales. Saps y Gupta inician discutiendo la falta de estudios controlados sobre tratamientos para el manejo del dolor abdominal en niños, habiendo sido realizados los existentes antes de la publicación de las primeras recomendaciones para ensayos clínicos de la Fundación de Roma ${ }^{17}$. Si bien las evidencias son escasas, los antiespasmódicos se consideran los tratamientos de primera línea para el tratamiento del dolor abdominal en niños ${ }^{17}$. El segundo tratamiento que se discute es la rifaximina. Solo dos estudios clínicos han sido realizados en niños, uno abierto y otro controlado con placebo, con resultados contradictorios ${ }^{18,19}$. En cuanto a los antidepresivos, los resultados de los únicos dos estudios controlados con tríciclicos (amitriptilina) no permiten establecer una recomendación para su uso. En forma similar, existen dos estudios con citalopram cuyos resultados son contradictorios ${ }^{20}$. Con relación a la ciproheptadina, un antihistamínico con propiedades anticolinérgicas y antiserotoninérgicas, ha sido analizada de manera retrospectiva en dispepsia funcional en un estudio prospectivo y dos retrospectivos, también con resultados mixtos, pero un estudio controlado en dolor abdominal funcional mostró su eficacia frente al placebo en la mejoría del dolor abdominal $^{21}$. En cuanto a los probióticos, la revisión de Saps y Gupta concluye que algunos parecen mejorar la frecuencia e intensidad del dolor abdominal, pero se requiere determinar las cepas y las dosis requeridas ${ }^{17}$. En resumen, las evidencias de los tratamientos farmacológicos son escasas y se requieren estudios controlados de tratamientos para el tratamiento del dolor abdominal en niños.

Con este número finalizamos el primer año exitoso de NeuroGastroLATAM Reviews, un proyecto que parecía imposible. No podemos concluir sin agradecer la labor del Dr. Luis Soifer como coeditor en jefe desde el inicio de la revista, a quien deseamos muchos éxitos en sus nuevos proyectos, y dar la más cordial bienvenida al Dr. Miguel Saps como nuevo coeditor en jefe. El Dr. Saps, un uruguayo afincado ahora en la Florida, es ün reconocido investigador a nivel mundial ê el campo de los trastornos funcionales găstrointestinales en niños. Su labor editorial fortalecerá el área pediátrica de la neurogâstroenterología en nuestra revista.

Estamos seguros de que los próximos años serán de crecimiento para NeuroGastroLATAM Reviews como medio de actualizaciốn para los interesados en la especialidad, no solo en Latinoamérica sino a nivel global.

\section{BIBLIOGRAFÍA}

1. Conners EE, Vinetz JM, Weeks JR, Brouwer KC. A global systematic review of Chagas disease prevalence among migrants. Ecta Trop. 2016;156:68-78.

2. Sguassero Y, Cuesta CB, Roberts KN, Hicks E, Comandé D, Ciapponi A, et al. Course of chronic Trypanosoma cruzi infection after treatment based on parasitological and serological tests: A systematic review of follow-up studies. PLoS One. 2015;10:e0139363.

3. Bilder CR, Goin JC. Gastrointestinal involvement in Chagas disease. NeuroGastroLatam Rev. 2017;1:168-179.

4. Adad SJ, Etchebehere RM, Jammal AA. Blood vessels in ganglia in human esophagus might explain the higher frequency of megaesophagus compared with megacolon. Rev Inst Med Trop Sao Paulo. 2014;56:529-32.

5. Jabari S, de Oliveira EC, Brehmer A, da Silveira AB. Chagasic megàcolon: enteric neurons and related structures. Histochem Cell Biol. 2014;142:235-44

6. Wallukat G, Munoz Saravia SG, Haberland A, Bartel S, Araujo R, Văłda $\mathrm{G}$, et al. Distinct patterns of autoantibodies against G-protein-coupled receptors in Chagas' cardiomyopathy and megacolon. Their potential impact for early risk assessment in asymptomatic Chagas' patients. J Am Coll Cardiol. 2010;55:463-8.

7. Corazziari ES. Gluten sensitivity and irritable bowel syndrome. NeuroGastroLatam Rev. 2017;1:180-186.

8. De Giorgio R, Volta U, Gibson PR. Sensitivity to wheat, gluten and FODMAPs in IBS: facts or fiction? Gut. 2016;65:169-78.

9. Skodje GI, Sarna VK, Minelle IH, Rolfsen KL, Muir JG, Gibson PR, et al. Fructan, rather than gluten, induces symptoms in patients with self-reported non-celiac gluten sensitivity. Gastroenterology. 2018;154:529-39. e2. 
10. Molina-Infante J, Serra J, Fernandez-Banares F, Mearin F. The low-FODMAP diet for irritable bowel syndrome: Lights and shadows. Gastroenterol Hepatol. 2016;39:55-65.

11. Solé L. Trastornos funcionales de la evacuación. Una revisión. NeuroGastroLatam Rev. 2017;1:187-205.

12. Rao SS, Bharucha AE, Chiarioni G, Felt-Bersma R, Knowles C, Malcolm A, et al. Anorectal disorders. Gastroenterology. 2016 Mar 25. [Epub ahead of print].

13. Martin-Martin GP, Garcia-Armengol J, Roig-Vila JV, Espí-Macías A, Martínez-Sanjuán V, Mínguez-Pérez M, et al. Magnetic resonance de-graphy versus videodefecography in the study of obstructed defecation syndrome: Is videodefecography still the test of choice after 50 years? Tech Coloproctol. 2017;21:795-802.

14. Ramage L, Simillis C, Yen C, Lutterodt C, Qiu S, Tan E, et al. Magnetic resonance defecography versus clinical examination and fluoroscopy: a systematic review and meta-analysis. Tech Coloproctol. 2017;21:915-27.

15. Wong RK, Drossman DA, Bharucha AE, Rao SS, Wald A, Morris CB, et al. The digital rectal examination: a multicenter survey of physicians' and students' perceptions and practice patterns. Am J Gastroenterol. 2012;107:1157-63.
16. Nensi A, Chande N. A survey of digital rectal examination training in Canadian medical schools. Can J Gastroenterol. 2012;26:441-4.

17. Saps M, Gupta SR. Functional abdominal pain disorders in children: An update on treatment. NeuroGastroLatam Rev. 2017;1:206-214.

18. Scarpellini E, Giorgio V, Gabrielli M, Filoni S, Vitale G, Tortora A, et al. Rifaximin treatment for small intestinal bacterial overgrowth in children with irritable bowel syndrome. Eur Rev Med Pharmacol Sci. 2013;17:1314-20.

19. Collins BS, Lin HC. Double-blind, placebo-controlled antibiotic treatment study of small intestinal bacterial overgrowth in children with chronic abdominal pain. J Pediatr Gastroenterol Nutr. 2011;52: 382-6.

20. Cooper TE, Heathcote LC, Clinch J, Gold JI, Howard R, Lord SM, et al. Antidepressants for chronic non-cancer pain in children and adelescents. Cochrane Database Syst Rev. 2017;8:CD012535.

21. Sadeghian M, Farahmand F, Fallahi GH, Abbasi A. Cyproheptadine for the treatment of functional abdominal pain in childhood: a doubleblinded randomized placebo-controlled trial. Minerva Pediatr. 2008;60:1367-74 